\title{
Comprehensive study of non touch enable password system
}

\author{
Monica Varia ${ }^{1}$, Hardik Modi ${ }^{2}$ \\ M.Tech Student, E.C Department, Charotar University of Science and Technology, Changa, India ${ }^{1}$ \\ Assistant Professor, E.C Department, Charotar University of Science and Technology, Changa, India ${ }^{2}$
}

\begin{abstract}
Since computer has been invented, people are using many devices to interact with computer and devices such as tablets, smart phones, etc. In the current age of technology, the user is expected to touch or non touch the machine interface to give input. Hand Gesture, where natural hand or red tape is used to communicate without touching machine interface. It gives a feeling of natural way of interacting with the devices. This paper presents the techniques used to interact with machine interface. Different non touch enable technologies through which user can give input to the particular system. Also introduces to the red color detection technology which is cost effective. This technology can be used in real time applications for password system in the areas like ATM, Jewellers Shops, Home Security, etc. This can be an attempt to replace stylus and keyboards with the natural finger without touching the screen.
\end{abstract}

Keywords: Hand Gesture; Non Touch Enable Technologies; Red Color Detection; ATM; Jewellers Shops; Home Security

\section{INTRODUCTION}

Communication using hand gestures exists in all for data entry and storage purpose. Still to the present, civilizations since old times. It is potentially a very natural Keyboards remain central to human-computer and useful modality for human machine interaction. The communication even as mobile devices such as smart use of hand gesture, as an interface between machine and phones and tablets adapt the keyboard as an optional human has always been a very attractive topic to be talk about. It has been applied to many applications using different techniques since few decades. Until now, touch based recognition on devices or sensors are used for interaction purpose. These sensor-glove based method obstructs the ease and naturalness with which humans interact with computers. This has led to an increased curiosity in the visual approach. These days, instruments that are coming to the market is enabled with the latest features. Such as in Tablets, Apple ipads, Samsung Galaxy, etc. Hand is the easiest way to show the expressions. Also that we can move it in any directions. The paper discusses the implementation of touch and non touch enable technologies. [1]

\section{BACKGROUND}

The first Computer was invented in 1822.[9] Since then, different types of techniques are being used to

interact with the computer and devices such as Smart phones, Tablets, etc.

\section{A. Keyboard}

The ancestral origin of all key-based text entry devices are typewriters, while the origin of computer keyboard as a device for electromechanical data entry and communication was largely from the two devices: teleprinters (or teletypes) and keypunches. [10] Fig.1 shows the computer keyboard typing technology. During 1870s, teleprinter-like devices were used to concurrently type and transmit stock market text data from the keyboard across telegraph lines to stock ticker machines to be immediately copied and displayed onto ticker tape. The keypunch devices having Keyboards played an vital role virtual, touch screen-based means of data entry. [10]

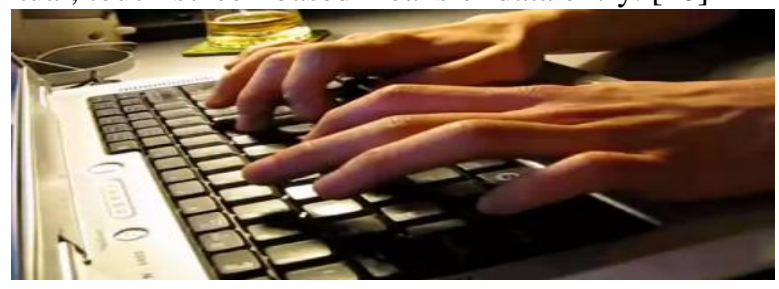

Fig. 1. Computer keyboard typing technology [11]

\section{B. Touchpad}

The Apollo desktop computers were equipped with a touchpad on the keyboard by 1982 . Touch pads began to be initiated in laptops during 1990s. [12] Fig.2 shows the touchpad technology.

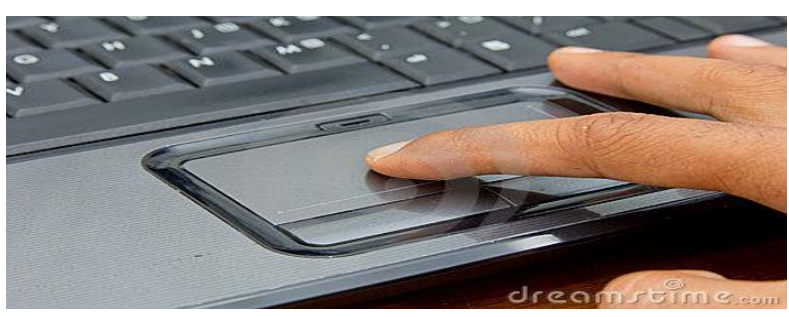

Fig. 2. Touch pad technology [13]

A touchpad/ or trackpad is a pointing device featuring a tactile sensor, a specialized surface that can translate the motion and position of a user's fingers to a relative position on the operating system that is outputted to the screen. Touch pads are a common feature of laptop computers, and are also used as a substitute for a mouse where desk space is scarce. [12] 


\section{Stylus}

A stylus is one type of a writing utensil, or a small tool for some other form of marking or shaping. [14] Forsline and Pederson came up with a stylus to use a pen kind e-stick to draw or write on a computer screen which changes the world of communication with the computers. [1] It can also be a computer accessory that is used to assist in navigating or providing more precision when using touch screens. [14] Fig.3 shows stylus technology.

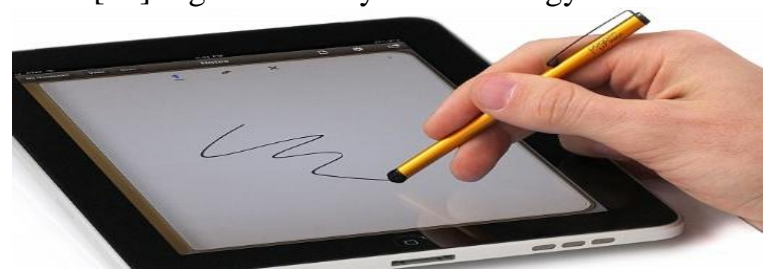

Fig. 3. Stylus technology[15]

\section{Non touch enable}

Today's the era of non touch enabled technology. Non touch enabled technology is the one in which user is expected to give input without touching machine interface. [1]User can also give input through any color tape on the finger. In Non touch enabled technology, mainly Hand Gesture is used to interact with the machines. Gestures are a form of non verbal communication which is in trend since humans born. Finger for non touch enabled technology is shown in fig. 4 .

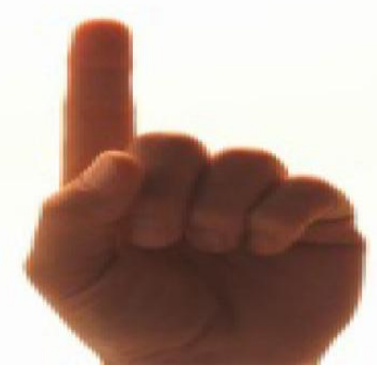

Fig. 4. Finger for non touch enable technology[1]

\section{VIRTUAL PASSWORD}

The use of passwords is known to be ancient. A password is used for user authentication to prove identity or access approval to gain access to a resource, which should be kept secret from those who don't have permission to access. [17-18]

Gesture recognition might not sound unfamiliar to the humans who are waiting to interact with the machines through normal body gestures. And non touch enable technology is such kind of.

Virtual keypads used to enter password may be used in some cases to reduce the risk of keystroke logging. It is more difficult for malware to monitor the display and mouse to obtain the data entered via virtual keypad. [1718] Virtual Password is shown in fig.5.

Yet, it is possible by recording screenshots at regular intervals or upon each mouse click.

Copyright to IJARCCE



Fig. 5. Virtual Password [19]

\section{WHY NEED VIRTUAL PASSWORD?}

As for the input of password, keypad is needed. So, it is a waste of money for the places where the keyboards with the devices which is only used to enter the password. Thus, to reduce the hardware cost, virtual password can be used.

It also comes along with the other advantages such as it does not require any space to place the keypad to enter the numbers. Also, it does not require sensors to interact and accuracy is good enough.

\section{DIFFERENCE BETWEEN TOUCH AND NON TOUCH ENABLED TECHNOLOGIES}

Touch enabled: In Touch Enabled Technology, input is given by touching the device.

\section{- $\quad$ Non touch enabled: In Non Touch Enabled} Technology, input is given by non touching the device.

\section{TYPES OF NON TOUCH ENABLED TECHNOLOGIES}

The different types of non touch enabled technologies used to interact with the computers are as follows:

\section{- $\quad$ Gesture Recognition}

Gesture recognition is a topic in computer science and language technology with the purpose of inferring human gestures via mathematical algorithms. Gestures can derive from any bodily motion or state but commonly originate from the face or hand. Current focuses in the field include emotion recognition from the face and hand gesture recognition. Gesture recognition can be seen as a way for computers to begin to understand human body language. Gesture recognition allows humans to communicate with the machine (HMI) and interact naturally without any mechanical devices. [20]



position

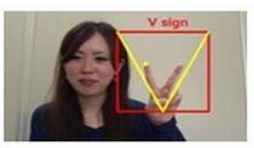

shape

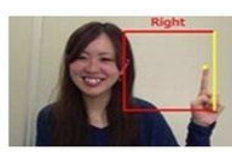

motion
Fig. 6. Gesture Recognition[21]

Fig 6. shows the example of gesture recognition. In first figure, index finger is shown that indicates point for position. In second figure, $\mathrm{v}$ sign is shown that indicates 
shape. And at the last, figure shows index finger at the right side which indicates motion.

\section{$\bullet$}

Color Detection

In color detection can be of various colors. To detect an object, color detection method can be used. In that any color can be used. We can consider red color. In red color tap technique, finger wrapped with the red color tap is to be detected. As the finger moves on the locations of the different numbers, that particular number is detected. This similar way is used to detect numbers and enter the password. In this technique, it detects the position of the finger and the centroid of red color part.

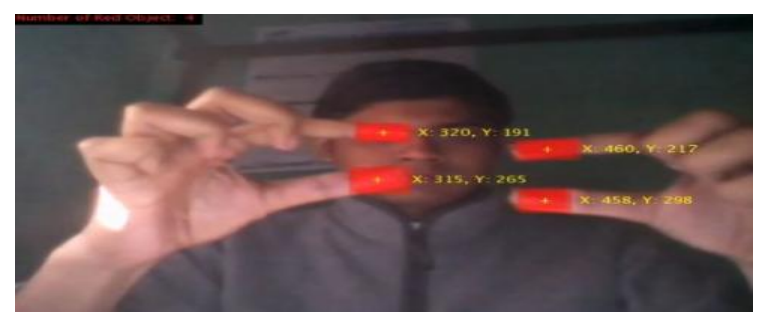

Fig. 7. Red Color Detection[22]

Fig 7. shows an example of red color technology. A person with his fingers with red color tap is detected. In the similar way, one can use the red color tap wrapped on the fingers and that will be used to detect the particular number on which the finger is kept. This way the digits of password can be entered. It can also be used for other devices.

\section{FEATURES OF NON TOUCH ENABLE TECHNOLOGY}

- Cost effective

- $\quad$ Replacement of keyboard

- $\quad$ Space requirement is nil

- $\quad$ Speed increases

\section{DESIGN TECHNOLOGY FOR NON TOUCH ENABLED SYSTEM}

For the purpose of virtual password to be available, the only thing needed is camera. Rest is the display screen of the device on which keypad can be displayed for the entering of the digits for password.

To design virtual password system, frame of the particular area is to be taken on which further process is done. Few functions are operated on the frame such as morphological, segmentation, and so forth.

Morphological image processing is a collection of nonlinear operations related to the shape or morphology of features in an image. These morphological operations rely on the relative ordering of pixel values and not on their numerical values. They are more widely suited for the process of binary images.[23] The main morphological functions are: stem, word-form, $\mathrm{x}$-form.[24]
In image segmentation, it is the process of partitioning a digital image into multiple segments that are make the set of pixels. This will change the image into more meaningful and easier for the analyse. [25]

Further, color image processing has to be done. By all the applications of processing needed finally the password is entered through the means of virtual keypad that is displayed and it is checked out with the one stored in the computer database.

\section{Application}

This technology of Non Touch Enabled can be used in the Real Time Applications like ATM machine, Jewellers Shops, Home Security, Mobile phones, etc. This can be used to replace keyboards to give input with the natural finger with the Red Color Tap without touching the screen.

\section{CONCLUSION AND FUTURE SCOPES}

In this paper, different technologies are described to provide the input to the system. Here, briefly color detection technology is discussed to enter the password in real time without use of Keyboard. Many existing systems available for commercial purposes like ATM, Jewellery Shops, Home Security, etc use keyboards to enter the password but non touch enable is the technology where no keyboard is used, would be an advantage to reduce the hardware cost and it is easy to use.

The non touch method provides a natural human system interaction in such a way that it does not require keyboard for input. This paper discusses the review of all the technologies that are being used till yet to enter the input to the systems. Meanwhile, it also describes about the password that is used since ancient times for the privacy of the valuable resources and to keep them safe. Virtual password is the one that can replace keyboard from the commercial places for the entering of password.

We can use any color Tap. We can also do this using normal finger tip without using any Color Tap.

It can be developed in plain background as well as in noisy background. That will provide much more robustness to the system developed.

This system will be Cost Effective. As it will replace all the keyboards it also needs less space to acquire the system.

\section{ACKNOWLEDGMENT}

We would like to thank the Charotar University of Science $\&$ Technology for its constant support all the way through our work.

\section{REFERENCES}

[1] Chaudhary, Ankit. "Natural Computing: Finger-Stylus for Non Touch-Enable Systems." arXiv preprint arXiv:1409.3554 (2014).

[2] Hagara, M.; Pucik, J., "Fingertip detection for virtual keyboard based on camera," Radioelektronika (RADIOELEKTRONIKA), 2013 23rd International Conference, vol., no., pp.356,360, 16-17 April 2013. 
[3] Jong-Min Kim; Woong-Ki Lee, "Hand Shape Recognition Using Fingertips," Fuzzy Systems and Knowledge Discovery, 2008. FSKD '08. Fifth International Conference on, vol.4, no., pp.44,48, 18-20 Oct. 2008

[4] Raheja, J.L.; Chaudhary, A.; Singal, K., "Tracking of Fingertips and Centers of Palm Using KINECT," Computational Intelligence, Modelling and Simulation (CIMSiM), 2011 Third International Conference on, vol., no., pp.248,252, 20-22 Sept. 2011

[5] Bragatto, T.A.C.; Ruas, G.I.S.; Lamar, M.V., "Real-time video based finger spelling recognition system using low computational complexity Artificial Neural Networks," Telecommunications Symposium, 2006 International, vol., no., pp.393,397, 3-6 Sept. 2006

[6] Sudderth, E.B.; Mandel, M.I.; Freeman, W.T.; Willsky, A.S., "Visual Hand Tracking Using Nonparametric Belief Propagation," Computer Vision and Pattern Recognition Workshop, 2004. CVPRW '04. Conference on, vol., no., pp.189,189, 27-02 June 2004

[7] Tu, J.; Huang, T.; Hai Tao, "Face as mouse through visual face tracking," Computer and Robot Vision, 2005. Proceedings. The 2nd Canadian Conference on , vol., no., pp.339,346, 9-11 May 2005

[8] Chaudhary, A.; Gupta, A., "Automated switching system for skin pixel segmentation in varied lighting," Mechatronics and Machine Vision in Practice (M2VIP), 2012 19th International Conference, vol., no., pp.26,31, 28-30 Nov. 2012

[9] Computer Technology Available :http://www.computerhope.com/issues/ch000984.htm

[10] Keyboard Technology Available : http://en.wikipedia.org/wiki/Computer_keyboard

[11] Keyboard Technology Available :http://en.wikipedia.org/wiki/Computer_keyboard\#mediaviewer/File :Keyboard typing.png

[12] Touchpad Technology Available :http://en.wikipedia.org/wiki/Touchpad

[13] Touchpad Technology Available :http://www.dreamstime.com/royalty-free-stock-image-fingernotebook-touchpad-image20144696

[14] Stylus Technology Available :http://en.wikipedia.org/wiki/Stylus

[15] Stylus Technology Available :http://thenextweb.com/apple/2011/02/26/5-stylus-pens-andbrushes-perfect-for-ipad-artists/

[16] Non Touch Enabled Technology Available :-http://en.wikipedia. org/wiki/List_of_gestures

[17] Password Available :http://en. wikipedia. org/wiki/password

[18] Virtual Password Available :http://en.wikipedia.org/wiki/virtual_keyboard

[19] Virtual Password Available :http://www.ebeijing.gov.cn/Life/shopping/ATMInBeijing/t914198. htm

[20] Gesture Detection Available :http://en.wikipedia.org/wiki/Gesture_recognition

[21] Gesture Detection available :http://www.33rdsquare.com/2012/05/omron- may-bring-handgesture.html

[22] Red Color Detection :http://arindambose.com/blog/?p=72

[23] Morphological Functions Available :https://www.cs.auckland.ac.nz/courses/compsci773s1c/lectures/Ima geProcessing-html/topic4.htm

[24] Morphological Functions Available :http://www.phon.ucl.ac.uk/home/dick/enc2010/articles/morpholog cal-function.htm

[25] Image Segmentation Available :http://en.wikipedia.org/wiki/Image_segmentation

[26] Jain, Anil K. Fundamentals of digital image processing. PrenticeHall, Inc., 1989

[27] Gonzalez, Rafael C., Richard E. Woods, and Steven L. Eddins. "Digital image processing using MATLAB." Upper Saddle River, N. J: Pearson Prentice Hall(2004). 